\title{
Immunoadsorption plasmapheresis for severe generalised myasthenia gravis
}

\author{
Motoki Ichikawa, Chang-Sung Koh, Yukiko Hata, Mari Tohyama, Takahisa Tsuno, \\ Atsushi Komiyama
}

\begin{abstract}
Two patients with severe generalised myasthenia gravis of childhood were successfully treated with immunoadsorption plasmapheresis. One patient was a 5 year old girl and the other was a 12 year old girl. A dramatic improvement in bulbar symptoms and generalised muscle weakness was achieved without any side effects. Plasma exchange may also yield clinical improvement for myasthenia gravis, but the treatment is accompanied by the risk of viral infections and hypersensitivity reactions. Immunoadsorption plasmapheresis is an effective treatment for severe generalised myasthenia gravis of childhood and is superior to plasma exchange with respect to safety.

(Arch Dis Child 1993; 69: 236-238)
\end{abstract}

Myasthenia gravis is an autoimmune disease characterised by muscle weakness due to impaired neuromuscular transmission. Circulating antibodies specific to acetylcholine receptor (AchR) of the neuromuscular junctions can be detected in many patients with myasthenia gravis. ${ }^{1}$ Antibodies to AchR are believed to cause the pathophysiology of myasthenia gravis. ${ }^{23}$

Anticholinesterase drugs, corticosteroids, and immunosuppressive drugs have been regarded as the main treatment strategies for myasthenia gravis. ${ }^{4}$ Immunoadsorption plasmapheresis has also been developed and its usefulness has been confirmed in adults. ${ }^{5-8} \mathrm{We}$ treated two children with severe generalised myasthenia gravis with immunoadsorption plasmapheresis. The patients showed a remarkable clinical improvement with treatment. We report here the effectiveness and safety of immunoadsorption plasmapheresis for severe generalised myasthenia gravis of childhood.

Shinshu University

School of Medicine,

Matsumoto, Japan,

Department of

Paediatrics

M Ichikawa

Y Hata

M Tohyama

T Tsuno

A Komiyama

Department of Medicine (Neurology) C-S Koh

Correspondence to: Dr Motoki Ichikawa,

Department of Paediatrics, Shinshu University School of Medicine, 3-1-1 Ashai, Medicine, 3-1-1 Ashai,
Matsumoto, 390 Japan.

Accepted 20 April 1993 days. Physical examination showed complete ophthalmoplegia, systemic muscle hypotonus, and respiratory insufficiency. The administration of edrophonium chloride gave a dramatic improvement in blepharoptosis Electromvographv showed decremental responses to repeated ulnar nerve stimulations. A diagnosis of generalised myasthenia gravis was made. Her serological data showed increased levels of immune complex and antinuclear antibodies. Antibodies to AchR were not detected by the biomedical laboratory (Tokyo). Computed tomography of her chest and thallium scintigraphy suggested thymic enlargement. Immediately after admission, femoral vein catheterisation was performed and two courses of immunoadsorption plasmapheresis using an immunoadsorbent column (Immusorba TR-350, Asahi Medical Company, Tokyo) were administered. The volume of plasma in the perfusion was 0.81 for each course. A remarkable improvement in the bulbar symptoms and generalised muscle weakness began on the second day of immunoadsorption plasmapheresis. We began treatment with prednisolone by mouth and anticholinesterase drugs. The ocular symptoms disappeared within one month (fig 1). There has been no recurrence of bulbar and ocular symptoms. After immunoadsorption plasmapheresis serum immunoglobulins decreased as follows: IgG from $15 \cdot 7$ to $6 \cdot 2 \mathrm{~g} /$, IgA from 2.3 to $1.4 \mathrm{~g} / \mathrm{l}$, and IgM from 2.2 to $1.0 \mathrm{~g} / \mathrm{l}$. Based on these data, $40-60 \%$ of serum immunoglobulins were adsorbed. Fibrinogen concentrations were decreased from 2.5 to 0.7 $\mathrm{g} / \mathrm{l}$. Neither clinical symptoms of immunodeficiency nor a tendency to bleed were shown.

\section{CASE 2}

A 12 year old girl was admitted to Shinshu University Hospital on 17 March 1992 with intermittent episodes of bilateral blepharoptosis, nasal voice, dysphagia, and muscle weakness of her arms for two months. Ten days before admission she had febrile episodes and a cough. A chest radiograph showed pneumonic infiltrations. Edrophonium chloride gave a dramatic improvement in blepharoptosis and electromyography showed decremental responses to repeated ulnar nerve stimulations. A diagnosis of generalised myasthenia gravis was made. Serum antibodies to AchR were not detected. She was treated with anticholinesterase drugs and antibiotics. Her blepharoptosis and pneumonia were improved within one week. Ten days after admission the generalised muscle weakness, dysphagia, dysarthria, and respiratory failure became worse. On 31 March mechanical ventilation was initiated for respiratory control. She also received two courses of immunoadsorption plasmapheresis for two days. The plasma 


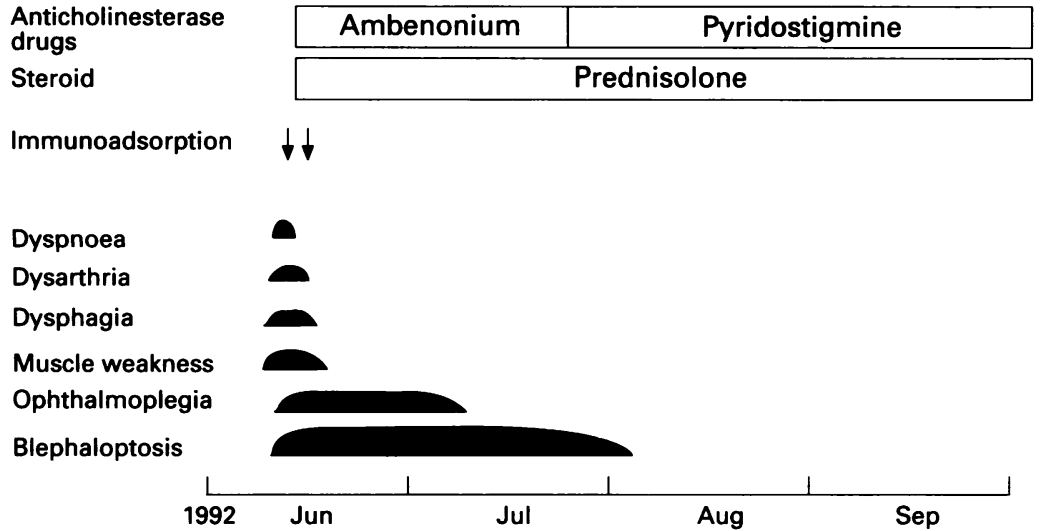

Figure 1 Clinical course of case 1.

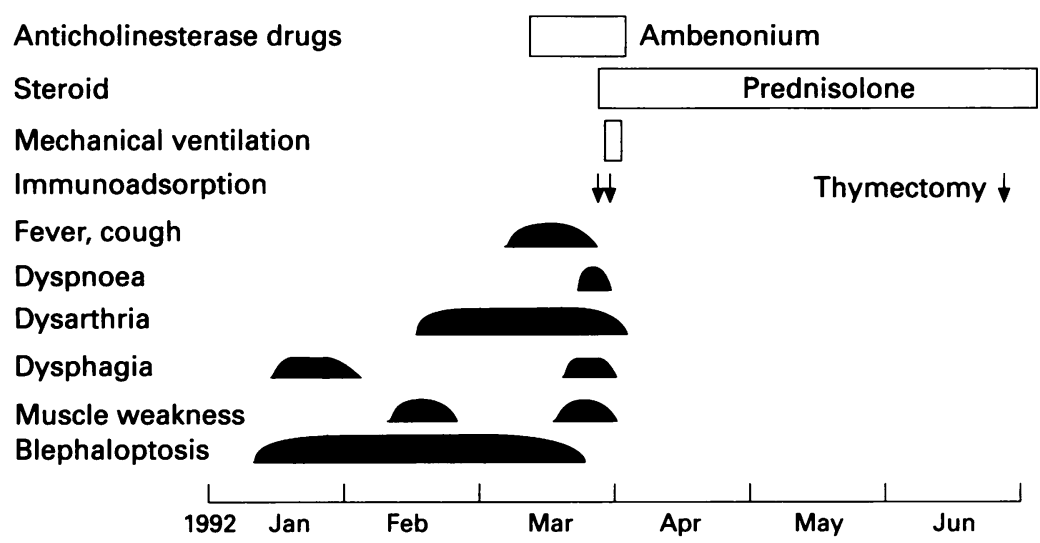

Figure 2 Clinical course of case 2.

volume of the perfusion was 1.81 for each course. On the second day of immunoadsorption plasmapheresis her dyspnoea and other bulbar symptoms improved. Mechanical ventilation was stopped and prednisolone administration begun (fig 2). Serum immunoglobulins and complements decreased as follows: IgG from 21.4 to $15.5 \mathrm{~g} / \mathrm{l}$, IgA from 4.4 to $3.0 \mathrm{~g} / \mathrm{l}$, IgM from 6.9 to $3.7 \mathrm{~g} / \mathrm{l}, \mathrm{C} 3$ from 1.7 to $1.2 \mathrm{~g} / \mathrm{l}$, and $\mathrm{C} 4$ from 0.32 to $0.16 \mathrm{~g} / 1$. Thus $30-50 \%$ of immunoglobulins and complements were adsorbed. Fibrinogen concentrations decreased from 3.3 to $1 \cdot 1 \mathrm{~g} / \mathrm{l}$. No clinical symptoms of immunodeficiency or tending to bleed were developed. On 24 June 1992 extended thymectomy was performed, and there has been no recurrence of the bulbar and ocular symptoms.

\section{Discussion}

We treated two children with severe generalised myasthenia gravis with immunoadsorption plasmapheresis and a remarkable improvement of bulbar symptoms and generalised muscle weakness was rapidly achieved. Remission was attained without any severe side effects from the treatment and early deterioration did not develop.

Myasthenia gravis is an autoimmune disease caused by antibodies to AchR. ${ }^{1}$ These antibodies are postulated to mediate inactivation or destruction of AchR. Antibodies to AchR are not necessarily detectable in all children with myasthenia gravis 9 ; antibodies to AchR were not found in these two children. It is possible that some AchR antibodies may not be detected by the assay used in our cases.

Plasma exchange is a method of removing antibodies to AchR from the circulation and produces a clinical improvement in myasthenia gravis in adults and children. ${ }^{1011}$ Plasma exchange loses important plasma components, however. In addition, there are risks of transfusion related infections (hepatitis or AIDS) and hypersensitivity reactions when fresh frozen plasma or plasma derivatives are used as replacement solutions.

Immunoadsorption plasmapheresis has been developed as a treatment for myasthenia gravis during the last few years. ${ }^{12}$ An immunoadsorbent TR-350 column consists of a synthetic resin embedded in a polyvinyl alcohol gel in which tryptophan is fixed as a ligand. Tryptophan is considered to be a selective ligand for antibodies to AchR because of the physicochemical interaction based on a hydrophobic bond. ${ }^{12}$ Several reports have shown that this new treatment can yield a clinical improvement in intractable generalised myasthenia gravis of adults without severe side effects. ${ }^{5-8}$ Generally $21-31 \%$ of IgG and $30-79 \%$ of antibodies to AchR are reduced with one session of immunoadsorption plasmapheresis. ${ }^{5-8}$ As in the cases reported here, antibodies to AchR are not always detectable in children with myasthenia gravis. Other antibodies against other AchR epitopes, which are not regularly measured, may play a part in childhood myasthenia gravis. Presumably these antibodies may also belong to the IgG fraction and be removed with immunoadsorption plasmapheresis. We have reported here the effectiveness and safety of immunoadsorption plasmapheresis for severe generalised myasthenia gravis of childhood. No side effects were seen. Immunoadsorption plasmapheresis may be superior to plasma exchange as a treatment of myasthenia gravis because plasma preparations are not used and there is less non-selective reduction of immunoglobulins.

Corticosteroids are widely used in the treatment of myasthenia gravis and are effective in most patients. ${ }^{413}$ Corticosteroids have several side effects such as cataracts, glaucoma, and diabetes mellitus, however. Of the side effects, the suppressive influence on bone growth is particularly important in children. In addition, an exacerbation in myasthenic weakness occurs in approximately $50 \%$ of patients treated with corticosteroids. ${ }^{9}$ Therefore corticosteroids should be used carefully and we should try to reduce the total amount used in the treatment of myasthenia gravis of childhood. In our patients a rapid improvement in bulbar symptoms and generalised muscle weakness was achieved and early deterioration was not observed. A rapid improvement in symptoms may shorten the duration of corticosteroid treatment. Thus long term repeated immunoadsorption plasmapheresis may be useful in the reduction of the total amount of corticosteroids.

Thymic enlargement was suggested in our first patient. Fortunately, this patient improved 
dramatically with immunoadsorption plasmapheresis treatment, corticosteroids, and anticholinesterase drugs. The benefit of thymectomy for patients with myasthenia gravis has been widely recognised. In childhood, however, the functions of the thymus are not fully understood and thymectomy in children with myasthenia gravis is controversial. Batocchi et al reported 59 children with myasthenia gravis and no patients under 9 years of age had a thymectomy. ${ }^{9}$

Our experience with those two patients indicates the effectiveness and safety of immunoadsorption plasmapheresis for severe generalised myasthenia gravis of childhood. Refractory myasthenia gravis of childhood and early onset generalised myasthenia gravis await better treatments. ${ }^{14} 15$ Further studies are expected to confirm that immunoadsorption plasmapheresis treatment may also be useful in these types of myasthenia gravis.

1 Drachman DB. Myasthenia gravis. N Engl F Med 1978; 298: $136-42$.

2 Seybold ME. Myasthenia gravis. A clinical and basic science review. $7 A M A$ 1983; 250: 2516-21.

3 Drachman DB. Myasthenia gravis. N Engl f Med 1978; 298: 186-93.

4 Keys PA, Blume RP. Therapeutic strategies for myasthenia gravis. DICP 1991; 25: 1101-8.
5 Sato T, Ishigaki Y, Komiya T, Tsuda H. Therapeutic immunoadsorption of acetylcholine receptor antibodies in myasthenia gravis. Ann NY Acad Sci 1988; 540: 554-6.

6 Passalacqua S, Splendiani G, Sturniolo A, et al. Immunosorbent treatment in myasthenia gravis. Prog Clin Biol Res 1990; 337: 285-8.

7 Hosokawa S, Oyamaguchi A. Safety, stability, and effectiveness of immunoadsorption under membrane plasmapheresis treatment for myasthenia gravis. ASAIO Trans 1990; 36: M207-8.

8 Splendiani G, Passalacqua S, Barbera G, et al. Myasthenia gravis (MG) treatment with immunoadsorbent columns. Biometerials, Artificial Cells, and Immobilization Biotechnology 1991; 19: 255-65.

9 Batocchi AP Evoli A Palmisani MT, Lo Monaco M, Bartoccioni M, Tonali P. Early-onset myasthenia gravis: clinical characteristics and response to therapy. Eur $f$ clinical characteristics and
Pediatr 1990; 150: $66-8$.

10 Pinching AJ, Peters DK, Davis JN. Remission of myasthenia gravis following plasma-exchange. Lancet 1976; ii: $1373-6$.

11 Snead OC, Kohaut EC, Oh SJ,. Bradley RJ. Plasmapheresis for myasthenic crisis in a young child. $\mathcal{F}$ Pediatr 1987; 110 740-2.

12 Sato T, Anno M, Arai K, Yamawaki N, Kuroda T, Inagaki $\mathrm{K}$. In vitro removal of anti-acetylcholine receptor antibodies with a new immunoadsorbent in sera from myasthenia ies with a new immunoadsorbent in sera from myasthenia
gravis patients. In: Atsumi K, Maekawa M, Oka K, eds. gravis patients. In: Atsumi K, Maekawa M, Oka K, eds.
Progress in Artificial Organs - 1983. Cleveland: ISAO Progress in Arrificial

13 Evoli A, Batocchi AP, Palmisani MT, Lo Monaco M, Tonali P. Long term results of corticosteroid therapy in patients with myasthenia gravis. Eur Neurol 1992; 32: 37-43.

14 Roach ES, Buono G, McLean WT, Weaver RG. Early-onset myasthenia gravis. I Pediatr 1986; 108: 193-7.

15 Sakano T, Hamasaki T, Kinoshita Y, Kihara M, Ueda K Treatment of refractory myasthenia gravis. Arch Dis Child 1989; 64: 1191-3. 